\title{
Comparison of analytical and difference sensitivity methods for structural damage identification
}

\author{
Jun Zhang, Kun Zhang \\ School of Civil Engineering, Dalian Jiaotong University, Dalian, 116028, China
}

\begin{abstract}
The sensitivity-based damage identification method receives much attention due to its ability to identify structural damages at element level. This paper aims to discuss the influence of two different deduction algorithms of sensitivity matrix, the analytical method and the numerical difference method, on the parameter identification results of structures with the same identification index. In order to compare the difference between the two sensitivity-based damage identification methods, the frequency-domain mode shape and the time-domain acceleration response information are used as the updating objectives, respectively. And then, the sensitivity matrices of the updating objectives to the same identification index, structural element stiffness parameters, are respectively derived based on the analytical method and the numerical difference method. Finally, a simply supported box girder bridge is used to illustrate the difference between the two sensitivity-based methods. Numerical simulations with initial model errors and measurement noise show the two types of methods both can accurately detect local damages and identify unknown initial model errors. The presence of random noise has some bad effect on the identified results of structural parameters, but the identified accuracy is still acceptable for normal noise level.
\end{abstract}

\section{Introduction}

Civil structures will inevitably produce damage accumulation in their use process. In order to ensure the normal use of structural functions and prevent the occurrence of dangerous engineering accidents, it is necessary to carry out health monitoring on the structures in service. Structural damage identification is the core of structural health monitoring systems[1,2]. In order to diagnose the local damages of the structures with the response information from the sensors, the parameters of the structure need to be inverted. The widely used methods of structural parameter inversion based on dynamic response are model correction methods. Because of the ability to identify the damage degree of element level, more and more attentions have been paid to the sensitivity-based model modification method[3,4]. The sensitivity matrix directly reflects the partial derivative relationship between the dynamic response of the structures and the stiffness parameters of the structure elements, and determines the quality of the parameter identification results. There are two main methods to construct the sensitivity matrix, who are the analytical method[5,6] and the numerical difference method[7,8]. Both of them are based on the finite element model. However, the former is derived from the dynamic formula, while the latter is based on the theory of the first-order Taylor expansion. They both can accurately identify the local damage information within the scope of online elasticity. However, due to the different formation of them, there is slightly difference between the two types of methods in recognition ability and application scope.

In this paper, the principle of the sensitivity methods taking modal shape and dynamic response acceleration as the updating objectives is briefly introduced, and then they are used to investigate the difference between the two sensitivity-based methods based on a numerical example of a simply supported box girder bridge of 30 meters in length.

\section{Basic theories}

\subsection{Sensitivity matrix in the analytical method}

\subsubsection{Sensitivity matrix based on modal shape}

Assuming that the structural damage is caused by the reduction of structural stiffness, which is independent of other structural characteristics, and that the change of stiffness is so small that it will not affect structural continuity and damping. Assuming that a small stiffness change occurs in the stiffness of an $n$-DOF system without considering damping, and the system response will change correspondingly

$$
\left[(\mathbf{K}+\Delta \mathbf{K})-\left(\omega_{i}^{2}+\Delta \omega_{i}^{2}\right) \mathbf{M}\right]\left(\boldsymbol{\Phi}_{i}+\Delta \boldsymbol{\Phi}_{i}\right)=0
$$

where $\mathbf{K}$ and $\mathbf{M}$ is $n \times n$ stiffness and mass matrices of the system; $\omega_{i}$ and $\boldsymbol{\Phi}_{i}$ is the $i$ th natural frequency and mode shape vector; $\Delta \omega_{i}$ and $\Delta \boldsymbol{\Phi}_{i}$ is the deviation of 
system response due $\Delta \mathbf{K}$. Neglecting second-order terms, eq.(1) can be written as

$$
\left(\mathbf{K}-\omega_{i}^{2} \mathbf{M}\right) \Delta \boldsymbol{\Phi}_{i}=\Delta \omega_{i}^{2} \mathbf{M} \Phi_{i}-\Delta \mathbf{K} \Phi_{i}
$$

$\Delta \boldsymbol{\Phi}_{i}$ can be express as a linear combination of mode shapes of the original system[9]

$$
\Delta \boldsymbol{\Phi}_{i}=\sum_{k=1}^{n} d_{i k} \mathbf{\Phi}_{k}
$$

where $d_{i k}$ is the coefficient of $i$ th linear combination corresponding to the $k$ th mode shapes. Substituting Eq.(3) into Eq.(2) and multiplying $\boldsymbol{\Phi}_{r}^{T}$ to both sides of Eq.(2)

$$
\sum_{k=1}^{n} d_{i k} \boldsymbol{\Phi}_{r}^{T}\left(\mathbf{K}-\omega_{i}^{2} \mathbf{M}\right) \boldsymbol{\Phi}_{k}=\Delta \omega_{i}^{2} \boldsymbol{\Phi}_{r}{ }^{T} \mathbf{M} \boldsymbol{\Phi}_{i}-\boldsymbol{\Phi}_{r}{ }^{T} \Delta \mathbf{K} \boldsymbol{\Phi}_{i}
$$

because of the orthogonality of mode shape, $d_{i k}$ can be written as

$$
d_{i r}=\frac{\boldsymbol{\Phi}_{r}{ }^{T} \Delta \mathbf{K} \boldsymbol{\Phi}_{i}}{\omega_{i}{ }^{2}-\omega_{r}{ }^{2}},(r \neq i)
$$

from Eq.(4) it can be found that $d_{r r}=0$. Therefore Eq.(3) can be rewritten as

$$
\Delta \boldsymbol{\Phi}_{i}=\sum_{\substack{r=1 \\ r \neq i}}^{n} \frac{\boldsymbol{\Phi}_{r}{ }^{T} \Delta \mathbf{K} \boldsymbol{\Phi}_{i}}{\omega_{i}{ }^{2}-\omega_{r}{ }^{2}} \cdot \boldsymbol{\Phi}_{r}
$$

within the scope of online elasticity, the reduction of structural stiffness $\Delta \mathbf{K}$ can be expressed as the summation of each elemental stiffness matrix multiplied by a damage coefficient

$$
\Delta \mathbf{K}=\sum_{k=1}^{n e l} \alpha_{k} \mathbf{K}_{k}=\sum_{k=1}^{n e l}\left(1+\Delta \alpha_{k}\right) \mathbf{K}_{k},\left(0 \leq \alpha_{k} \leq 1,-1 \leq \Delta \alpha_{k} \leq 0\right)
$$

where $\mathbf{K}_{k}$ and $\alpha_{k}$ is the $k$ th elemental stiffness matrix and its damage coefficient; nel is the total number of element. Substituting Eq.(7) into Eq.(6), and Eq.(6) can be rewritten as

$$
\Delta \boldsymbol{\Phi}_{i}=\sum_{k=1}^{n e l} \alpha_{k} \sum_{\substack{r=1 \\ r \neq i}}^{n} \frac{\boldsymbol{\Phi}_{r}{ }^{T} \mathbf{K}_{k} \boldsymbol{\Phi}_{i}}{\omega_{i}^{2}-\omega_{r}{ }^{2}} \cdot \boldsymbol{\Phi}_{r}=\mathbf{S}_{\boldsymbol{\Phi}_{i}} \cdot \boldsymbol{\alpha}
$$

where $\alpha=\left\{\begin{array}{llll}\alpha_{1} & \alpha_{2} & \ldots & \alpha_{n e l}\end{array}\right\}^{T}$, and the vector of sensitivity coefficients of the $i$ th mode shape $\mathbf{S}_{\Phi_{i}}$ can be written as

$$
\mathbf{S}_{\boldsymbol{\Phi}_{i}}(\boldsymbol{\alpha})=\left(\sum_{\substack{r=1 \\ r \neq i}}^{n} \frac{\boldsymbol{\Phi}_{r}{ }^{T} \mathbf{K}_{1} \boldsymbol{\Phi}_{i}}{\omega_{i}{ }^{2}-\omega_{r}{ }^{2}} \boldsymbol{\Phi}_{r} \quad \cdots \quad \sum_{\substack{r=1 \\ r \neq i}}^{n} \frac{\boldsymbol{\Phi}_{r}{ }^{T} \mathbf{K}_{n e l} \boldsymbol{\Phi}_{i}}{\omega_{i}{ }^{2}-\omega_{r}{ }^{2}} \boldsymbol{\Phi}_{r}\right)
$$

\subsubsection{Sensitivity matrix based on dynamic response}

The equations of motion of a $n$-DOF damped system can be expressed as

$$
\mathbf{M} \ddot{\mathbf{y}}(t)+\mathbf{C} \dot{\mathbf{y}}(t)+\mathbf{K y}(t)=\mathbf{L}(t) \cdot \mathbf{F}(t)
$$

where $\mathbf{C}$ is the damping matrix. According to Rayleigh damping theory, it can be expressed as $\mathbf{C}=$ $a_{1} \mathbf{M}+a_{2} \mathbf{K}$, and $a_{1}, a_{2}$ are the Rayleigh damping coefficient; $\mathbf{y}(t), \dot{\mathbf{y}}(t)$, and $\ddot{\mathbf{y}}(t)$ are, respectively, the displacement, velocity and acceleration response of the structure at time $t ; \mathbf{L}(t)$ is the mapping matrix relating the external excitation $\mathbf{F}(t)$ to the corresponding DOFs of the structure. Neglecting the load uncertainty, $\mathbf{L}(t)$ and $\mathbf{F}(t)$ must be fully known at any time.

Assuming the structural local damages are in the form of fractional change in the stiffness of an element.
Performing differentiation to both sides of the Eq.(10) with respect to the physical parameters of structures, we have

$$
\begin{aligned}
\mathbf{M} \frac{\partial \ddot{\mathbf{y}}(t)}{\partial \alpha_{k}}+\mathbf{C} \frac{\partial \dot{\mathbf{y}}(t)}{\partial \alpha_{k}}+\mathbf{K} \frac{\partial \mathbf{y}(t)}{\partial \alpha_{k}}=-\mathbf{K}_{k} \mathbf{y}(t)-a_{2} \mathbf{K}_{k} \dot{\mathbf{y}}(t) \\
\mathbf{S}_{\ddot{\mathbf{y}}(t)}=\left[\begin{array}{lll}
\frac{\partial \ddot{\mathbf{y}}(t)}{\partial \alpha_{1}} & \cdots & \frac{\partial \ddot{\mathbf{y}}(t)}{\partial \alpha_{n e l}}
\end{array}\right]
\end{aligned}
$$

where $\mathbf{S}_{\ddot{y}(t)}$ is the vector of sensitivity coefficients of the response acceleration $\ddot{\boldsymbol{y}}$ at time $t ; \frac{\partial \ddot{\boldsymbol{y}}(t)}{\partial \alpha_{k}}$ is the coefficient of $k$ th elemental stiffness parameter $\alpha_{k}$, which is calculated by numerical integration method from Eq.(11); $a_{2}$ represents the Rayleigh damping coefficient related to the stiffness matrix.

\subsection{Sensitivity matrix in the numerical difference method}

The difference sensitivity is based on the first-order Taylor expansion principle, also known as the first-order perturbation method. Setting a perturbation $p$ for stiffness parameter $\alpha_{k}$, then the response calculated by the finite element method will produce a variation value $\Delta \mathbf{R}$. So the sensitivity of the response can be expressed as the ratio between $\Delta \mathbf{R}$ and $p$

$$
\mathbf{S}=\left[\begin{array}{cccc}
\frac{\Delta \mathbf{R}_{1}}{p} & \frac{\Delta \mathbf{R}_{2}}{p} & \cdots & \frac{\Delta \mathbf{R}_{n e l}}{p}
\end{array}\right]
$$

where $\Delta \mathbf{R}_{k}=\mathbf{R}_{k}\left(\alpha_{k}+p\right)-\mathbf{R}_{k}\left(\alpha_{k}\right)$

\subsection{Iterative recognition algorithm based on response sensitivity}

After the dynamic indexes (modal shape, accelerations etc.) and their sensitivities with respect to the physical parameters of the structure are obtained, the next step is to find the vector of physical parameters by matching the calculated dynamic indexes and the measured dynamic indexes in a iterative optimization procedure. The basic theory of sensitivity-based recognition algorithm is that the response from field measurements can be expressed in a first-order Taylor expansion as

$$
\mathbf{R}^{m}=\mathbf{R}^{c}+\mathbf{S} \cdot \Delta \boldsymbol{\alpha}
$$

where $\mathbf{R}^{c}$ is the response from the finite element model; $\mathbf{S}$ is the sensitivity matrix. Eq.(14) can be rewritten as

$$
\Delta \mathbf{R}^{k}=\mathbf{S}^{k} \cdot \Delta \boldsymbol{\alpha}^{k+1},(k=0,1,2, \cdots)
$$

The response vector $\mathbf{R}^{c}$ and sensitivity matrix $S$ need to be updated continuously in order to complete the iteration of the algorithm, this is mainly because the damage detection equation is expressed in the form of first-order Taylor expansion, which will result in a non-linear relationship between $\Delta \mathbf{R}$ and $\Delta \alpha$ in Eq.(15) The error caused by this non-linearity can be reduced by successive iterative process. The flowchart of sensitivity-based damage identification method is shown in Fig.1. 


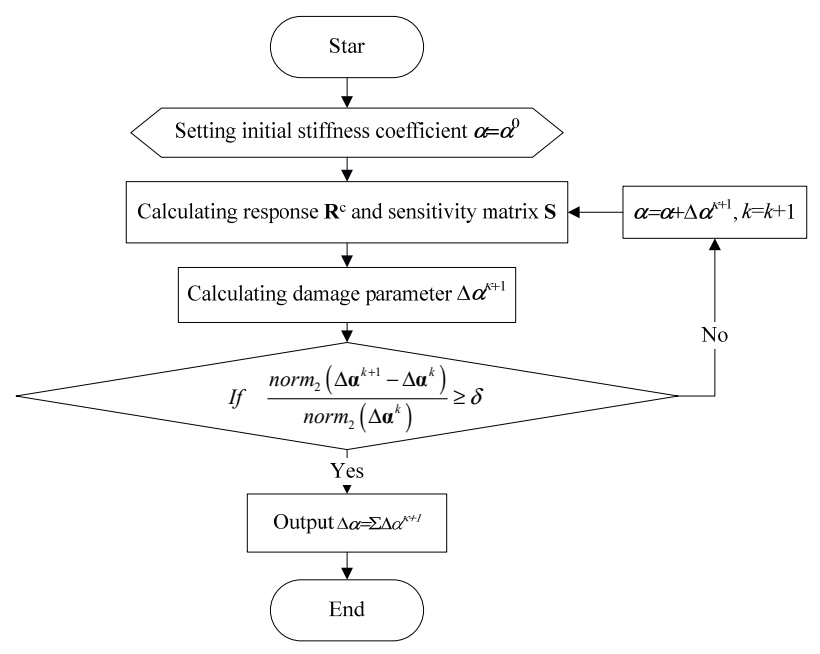

Fig.1. Flow chart of sensitivity-based damage identification algorithm

In Fig.1, the superscript $k$ is the iteration number. The pre-selected criterion $\delta$ is equal to $10^{-10}$ in this study. The iterative damage detection equation in Eq. (15) is solved by the adaptive Tikhonov regulation method [10]. Finally, the damage parameters can be obtained by $\Delta \boldsymbol{\alpha}=\Sigma \Delta \boldsymbol{\alpha}^{k+1}$.

\section{Numerical simulations}

A simply supported box girder bridge of 30 meters in length is numerically used to illustrate the differences between the two sensitivity methods. The finite element model of the bridge consists of 31 nodes and 30 plane Euler beam elements of 1 meter in length. The Young's modulus of structural material $E=3.45 \times 10^{10} \mathrm{~N} / \mathrm{m}^{2}$, the bending moment of inertia $I=12.752 \mathrm{~m}^{4}$, the poisson's ratio $\gamma=0.2$. According to the finite element method, the first eighth natural frequencies of the complete model are 18.43, 71.89, 160.49, 283.52, 439.88, 627.97, 845.45, $1089.03 \mathrm{~Hz}$. In order to compare the influence of different sensitivity matrix on the results of damage identification, a structural damage scenario is set up with the Young's modulus of the 12th, 17th, and 25th element reduced by $10 \%, 5 \%$ and $15 \%$. On the basis of these damages, a group of Gaussian white noise is generated randomly according to $1 \%$ of the value of the original structural stiffness parameter, and it is added to the stiffness parameters of the structure together with the damage parameters to simulate the model errors.

\subsection{Identification based on modal shape}

Fig. 2 and Fig. 3 illustrate the sensitivity diagram of the analytical method and the numerical difference method of the 1th and the 10th modal shapes of the intact structure under the environmental excitation. The letter $\mathrm{d}$ in the figure indicates the perturbation step size of element stiffness parameter in the difference method.

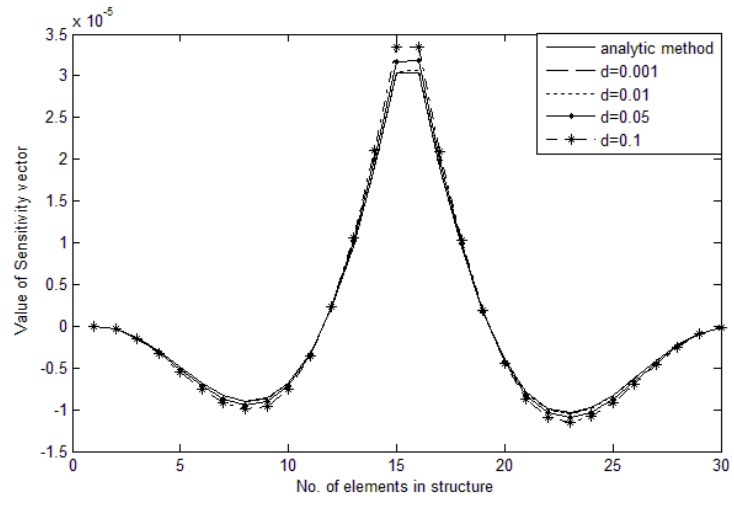

Fig.2. The fluctuation of the 1 th modal shape sensitivity

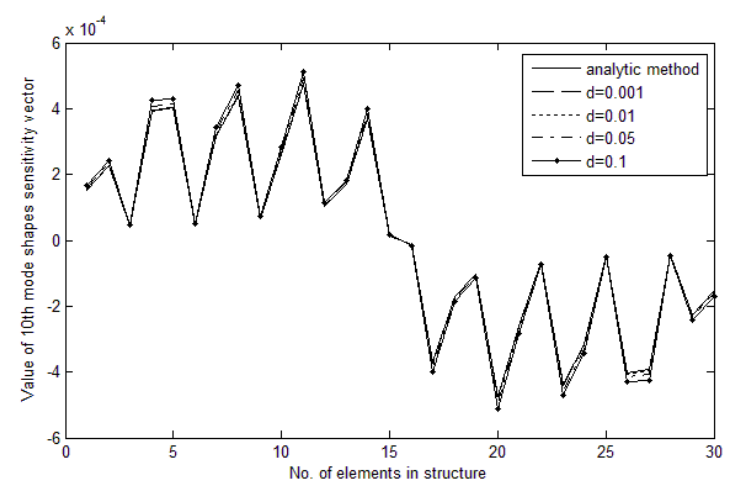

Fig. 3. The fluctuation of the10th modal shape sensitivity

It can be seen from the two figures that the wave forms of each type of sensitivity vector are very similar, but with the increase of the perturbation step size $d$, the amplitude difference in sensitivity vector between the difference and analytical methods increases when the step size exceeds 0.01 .

In order to explore the effects of different sensitivity methods based on modal shape on the damage identification results, the same damage scenario are identified according to the different sensitivity methods including the analytical method, and the difference method with $\mathrm{d}=0.001, \mathrm{~d}=0.01, \mathrm{~d}=0.05$ and $\mathrm{d}=0.1$ respectively. Table 1 shows the error 2-norm of damage identification results corresponding to different sensitivity method. The significant number after the decimal point is reserved for the fourth number only.

Table 1. Error 2-norm of damage identification results

\begin{tabular}{|c|c|c|c|c|}
\hline & Analytic method & $\mathrm{d}=0.001$ & $\mathrm{~d}=0.01$ & $\mathrm{~d}=0.05$ \\
\hline Determined & 0.4998 & 7.9257 & 7.1289 & - \\
\hline Over-determined & 0 & 0 & 0 & 0 \\
\hline
\end{tabular}

It can be seen from Table 1 that the difference parameter perturbation will have a significant impact on the damage identification results when the identification equations are determined. However, the negative effect will be reduced when the identification equations are strict over-determined. For the over-determined case, both the analytic method and the difference method with d- $0.001, \mathrm{~d}-0.01, \mathrm{~d}-0.05$ are both capable of accurately identify structural damages, but the difference method with d-0.1 fails. Moreover, the identification results with 
small error can be obtained by the analytical sensitivity method even with the determined identification equations. Because of the incompleteness of measuring points and the lack of high-order frequency-domain information, the identification equations based on frequency-domain information can only achieve the damage identification with the minimum number of equations. This has a certain engineering significance.

In general, it is difficult to get very accurate identification results with few identification equations. Therefore it is necessary to optimize the location of measuring points. The optimization method of measuring points is based on the correlation between sensitivity vectors. This method is similar to the modal assurance criterion of mode shapes. The specific optimization process can refer to the sensitivity vector correlation optimization method of time-domain information[11].

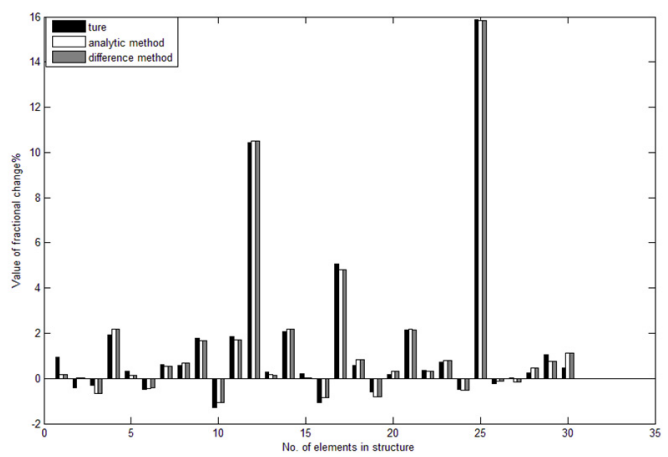

Fig.4. Damage identification results with 3\% measurement noise based on modal shape

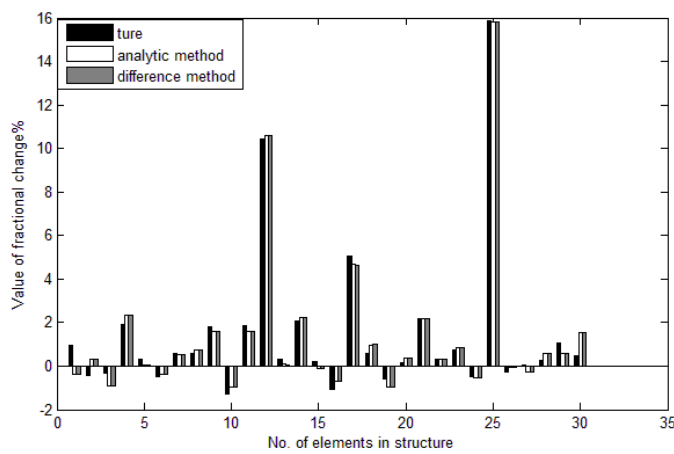

Fig.5. Damage identification results with 5\% measurement noise based on modal shape

Fig.4 and Fig.5 show the damage identification results of the analytical sensitivity method and the differential sensitivity method with $\mathrm{d}=0.01$ from the over-determined equations under the scenario of $3 \%$ and $5 \%$ measurement noise. It can be seen from these figures that there is not much difference between the analytical sensitivity method and the differential sensitivity method with over-determined equations. This indicates that both the analytical sensitivity method and the differential sensitivity method based on modal shapes can be used to identify structural damages with enough equations and suitable perturbation step size, and they have good noise robustness.

\subsection{Identification method based on dynamic acceleration}

Fig.6 shows the sensitivity vector of the midspan position of the bridge structure under a $10^{5} \mathrm{~N}$ moving concentrated force at a certain time. Because the position of the force changes with time, therefore, it is necessary to consider the equivalent node load of the concentrated force calculated according to the position of the action point as the complete external excitation[12]. It can be seen from this figure that the sensitivity vectors of different methods based on time-domain information have the similar wave form, and the difference amplitude between the analytical sensitivity method and the differential sensitivity method also increases with the increase of the perturbation step size. The amplitude of sensitivity vector with $\mathrm{d}=0.001$ and $\mathrm{d}=0.01$ in the difference method is also very close to that of the analytical method.

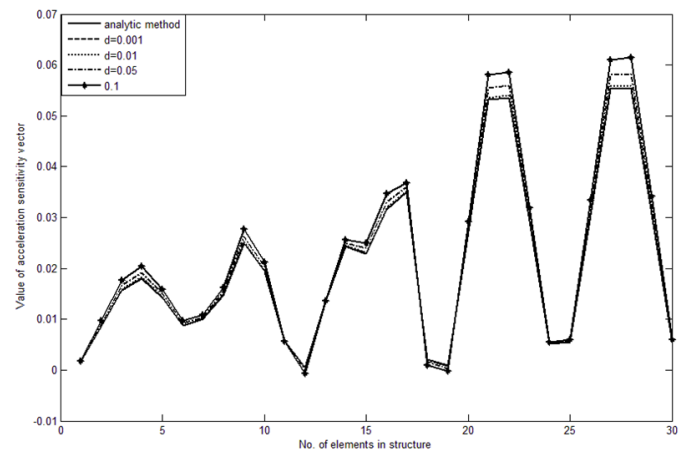

Fig.6. The fluctuation of response acceleration sensitivity

In terms of index parameters, the damage identification method based on time-domain information can get enough identification equations, and it is generally difficult to find the case that the equations are determined or underdetermined. Therefore, the damage identification of the whole structure can be realized by only using the acceleration information from the measuring point of the midspan position of the bridge structure. Fig.7, Fig.8 and Fig.9 show the damage identification results of the two sensitivity methods with noise-free, $3 \%$ and 5\% measurement noise, where the perturbation step size $d=0.01$ in the difference method.

It can be seen from Fig.7 that both the structural damages and initial model errors are accurately identified by using the two types of methods in the noise free case. Whereas, Fig.8 and Fig.9 illustrates the presence of random noise has some bad effect on the identified results of structural parameters, but the identified accuracy is still acceptable for this noise level. It may be concluded that measurement noise is the most important influencing factor, and measurements with a high accuracy are therefore needed for the identification method based on dynamical response. 


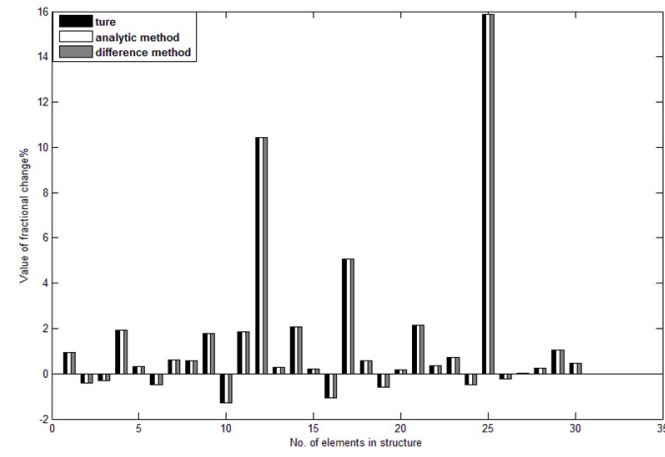

Fig. 7. Damage identification results without measurement noise based on dynamic responses

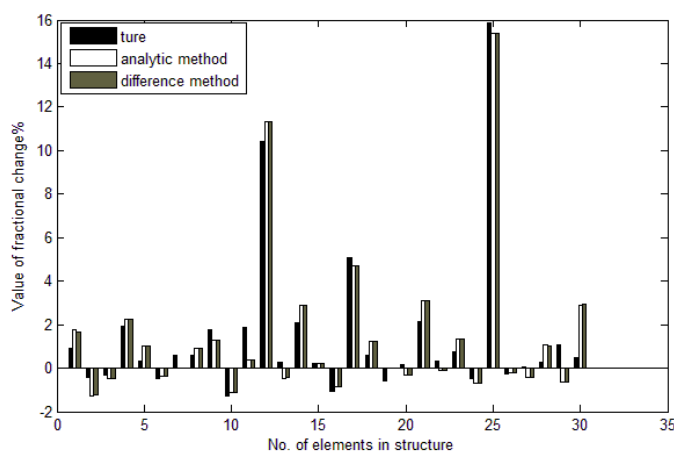

Fig. 8. Damage identification results with 3\% measurement noise based on dynamic responses

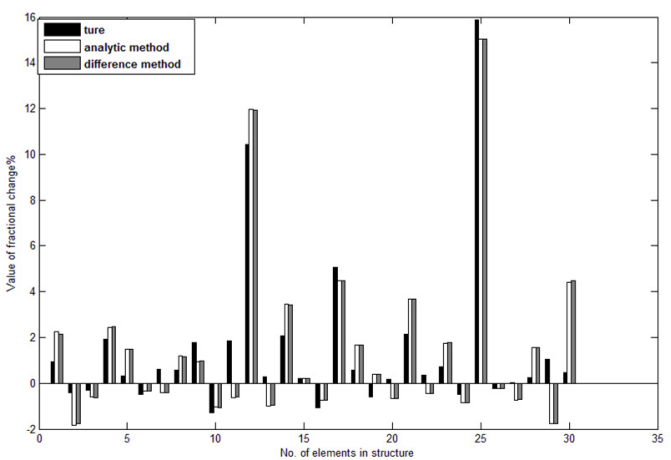

Fig. 9. Damage identification results with 5\% measurement noise based on dynamic responses

\section{Conclusion}

(1) No matter based on time-domain or frequency-domain parameters, each row vector in sensitivity matrix constructed by analytical method or numerical difference method has the same wave form, but the amplitude of fluctuation is slightly different. Both of them have the same convergence form and can get similar recognition results. In addition, the optimal combination of measurement points obtained by the same sensor optimization method based on sensitivity is the same.

(2) When the frequency-domain information is used as the target parameter of model modification, the number of identification equations is too small due to the incompleteness of measuring points and the difficulty of measuring high-order modal information, the identification result of analytical sensitivity method is more accurate and stable.

(3) Although the two sensitivity damage identification methods have the same convergence form in the identification process, the analytical method does not need to calculate the structural response repeatedly when constructing the sensitivity, which can save time for the high computation problem. It takes a lot of time to calculate the structural response repeatedly when using the numerical difference method to build the sensitivity. But the concept of numerical difference method is clear and the formula is simple. For the complex response derived information, the differential sensitivity method has a more extensive application prospect.

(4) Considering the stability of the recognition algorithm, the parameter perturbation value should not be more than 0.01 when using the numerical difference method to construct the sensitivity matrix.

\section{Acknowledgment}

Authors wishing to acknowledge financial supports from Research Foundation from the Education Department of Liaoning Province (JDL2019029), Science Foundation of Liaoning Province (20170540130), and Scientific Research Foundation for Introduced Talents of Dalian Jiaotong University.

\section{References}

1. G.W. Housner, L.A. Bergman, T.K. Caughey et al., J. Eng. Mech.-ASCE 123(1997)

2. S.W. Doebling, C.R. Farrar, M.B. Prime, Shock Vib. 30(1998)

3. A. Messina, E.J. Williams, T. Contrusi, J. Sound Vib. 216(1998)

4. S.S. Law, X.Y. Li, Z.R. Lu, J. Eng. Mech.-ASCE. 132(2006)

5. H.F. Lam, J. Kom, C.W. Wong, J. Sound Vib. 210(1998)

6. Y. Hui, S. S. Law, C.J. Ku, J. Sound Vib . 389(2017)

7. X.Y. Li, S.S. Law, Mech. Syst. Signal Pr. 24(2010)

8. S.S. Law, K. Zhang, Z.D. Duan, Eng. Struct. 32(2010)

9. R.L. Fox, M.P. Kapoor, AIAA J. 6(1988)

10. X.Y. Li, S.S. Law. Mech, Syst. Signal Process. 24(2010)

11. J. Wang, S.S. Law, Q.S. Yang, Mech. Syst. Signal Pr. 41(2013)

12. S.S. Law, J.Q. Bu, S.L. Chan, Eng. Struct. 26(2004) 